\title{
Post-mortem ultrasonography: a safer alternative to autopsies in COVID-19 deaths
}

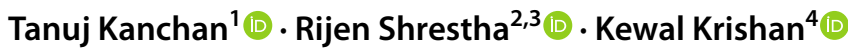

Received: 30 July 2020 / Accepted: 5 October 2020 / Published online: 31 October 2020

(c) Società Italiana di Ultrasonologia in Medicina e Biologia (SIUMB) 2020

\begin{abstract}
Ultrasonography is known to have many applications in the diagnoses of diseases, as well as in guiding medical practitioners through precise medical procedures. However, its use as a post-mortem radiographic modality has been limited. Postmortem ultrasonographic techniques are considered to be a safer alternative to high-risk post-mortem procedures, especially in infectious diseases. The present communication discusses the possibilities of using ultrasonography in post-mortem examinations in times of the ongoing COVID-19 pandemic to minimize the associated risk of SARS-CoV-2 infection of those working in mortuaries during full-body dissection in traditional autopsies. Post-mortem ultrasonography can be useful in reducing the extent of autopsies, thus decreasing the risk of exposure of forensic personnel.
\end{abstract}

Keywords COVID-19 pandemic $\cdot$ SARS-CoV-2 $\cdot$ Post-mortem examination · Ultrasonography $\cdot$ Safety of forensic personnel

SARS-CoV-2 infection has resulted in more than $28,637,952$ cases and about 917,417 deaths globally as of September 13, 2020 [1]. The infectious nature, as well as the high morbidity and mortality associated with the virus, has been a major concern for healthcare workers, including forensic pathologists, autopsy surgeons, and support staff in mortuaries. At a time when there is community spread of COVID-19, it is difficult to determine whether a deceased person who died of a natural or a non-natural cause, which necessities postmortem examination was COVID-19 positive or not. In the absence of post-mortem testing of the deceased, it is especially relevant to know a person's COVID-19 status prior to performing an autopsy. Therefore, it is suggested that in all cases, autopsy surgeons and support staff take all due precautions and strictly adhere to all the infection prevention

Kewal Krishan

gargkk@yahoo.com; kewalkrishan@pu.ac.in

1 Department of Forensic Medicine and Toxicology, All India Institute of Medical Sciences, Jodhpur, India

2 Department of Forensic Medicine, Tribhuvan University Institute of Medicine, Kathmandu, Nepal

3 Department of Anthropology, Panjab University, Chandigarh, India

4 Department of Anthropology (UGC Centre of Advanced Study), Panjab University, Sector-14, Chandigarh, India and control practices to safeguard themselves. The situation, however, may be much harsher in resource-limited settings [2], especially in view of the ill-equipped mortuaries and the limited availability of personal protective equipment in many parts of the world.

Numerous modifications to a complete autopsy examination have been recommended for minimizing the risk of infection during autopsies, including reduced manipulations of the body, performing in situ examinations as opposed to whole-block eviscerations, and avoiding embalming [3]. These measures have been relatively successful in preventing the spread of infection among forensic personnel but have raised concerns about missing vital information and misdiagnosing the cause of death. "Virtopsy", which uses primarily computed tomography (CT) and magnetic resonance imaging (MRI) in post-mortem examinations, has been suggested as an effective alternative to high-risk traditional autopsy to minimize the risk of transmission of SARS-CoV-2 during an autopsy. In the absence of virtopsy facilities, ultrasonography (USG) may be a cost-effective and safer alternative for post-mortem examinations [4]. Post-mortem USG can be useful in reducing the extent of autopsies, thereby decreasing the risk of infection of forensic personnel.

USG as a post-mortem imaging modality can help reduce the need for extensive autopsies in finding the cause of death. 
The conditions that can be noticed by post-mortem USG include cardiac hypertrophy, pericardial tamponade, aneurysm of the abdominal aorta, pleural effusions, subphrenic abscesses, ascites or intra-abdominal bleeding, liver metastasis, liver cirrhosis, fatty liver or fatty changes in the liver parenchyma, bile stones, renal cysts, diverticulum of the urinary bladder, hyperplasia of the prostate, myoma uteri, intracranial hemorrhage in infants, bone fractures, and foreign substances in mammae (implant) [5]. Lung USG (LUS) is considered to be a rapid, sensitive, and non-invasive bedside method for detecting several pulmonary pathologies, and its role in COVID-19 is being explored [6]. Some of the characteristic LUS findings in COVID-19 include thickening and irregularities of the pleural line, B lines, and a variety of consolidations. A lines have been described to appear during the recovery phase of COVID-19 [7]. "Echopsy", or ultrasonographic autopsy, is a modification of needle autopsy for the ultrasound-guided collection of tissue samples for further examination. Echopsy, too, is regarded as a feasible and reliable alternative to traditional autopsy [8]. The risk of infection is minimal with echopsy; hence, it is considered to be a safer technique of post-mortem examination in cases of infectious diseases, such as COVID-19.

The refrigeration and hardening of adipose tissue have been shown to affect the permeation and reflection of ultrasound [5]. In the case of refrigerated bodies, it is recommended to perform USG 1-2 $\mathrm{h}$ after the body has been removed from the cold chamber. One of the major limitations to the use of USG in post-mortem examinations is the obliteration of the view caused by decomposition gases. Uchigasaki recommends the use of USG within 4-5 days after death [5]. In view of its limitations, Charlier et al. recommend the use of USG only for the estimation of facial tissue thickness and for guided biopsies [9].

Although USG may not be useful in post-mortem examinations of all medico-legal cases, it can certainly assist in validating findings from medical records and in reducing the need for autopsy in such cases. Similarly, for cases with a known history of violence, USG can assist in localizing the area of investigation, thus reducing the need for extensive dissection. Besides, echopsy or post-mortem USG can be a useful adjunct to the traditional autopsy examinations and can assist in reducing the extent of the dissection required. Thus, USG can be judiciously employed as a safer technique for post-mortem examinations.

There have been major advances in the use of post-mortem imaging as an adjunct to traditional autopsy. However, these advances have been limited to the use of CT. Post-mortem USG can be especially useful in addressing the health and safety concerns of the forensic personnel involved in autopsy work, shielding them from unwarranted exposure to SARS-CoV-2 during the COVID-19 pandemic, especially in low-resource settings.
Acknowledgements Kewal Krishan is supported by a DST PURSE grant and the UGC Centre of Advanced Study (CAS II) awarded to the Department of Anthropology, Panjab University, Chandigarh, India.

Author contributions TK: conceptualization, writing, review and editing, final approval and supervising the work; RS: writing original draft, review and editing, final approval; KK: writing, review and editing, final approval and supervising the work.

Funding None.

Data availability The data and the materials are available with one of the authors (RS).

\section{Compliance with ethical standards}

Conflict of interests The authors declare that there are no competing interests regarding this manuscript.

Ethics approval and consent to participate Not applicable in this case.

Consent for publication All the authors approved the final version of the manuscript and provided consent for publication in the journal.

\section{References}

1. WHO, World Health Organization (2020) Coronavirus disease (COVID-2019) dashboard. https://covid19.who.int. Accessed 13 Sep 2020

2. Kanchan T, Saraf A, Misra S (2020) COVID-19 outbreak: a testing time for medicolegal facilities in India. J Indian Acad Forensic Med 42:1-2. https://doi.org/10.5958/0974-0848.2020.00001.9

3. Finegan O, Fonseca S, Pierre GH, Mendez MDM, Gonzalez JR, Tidball-Binz M et al (2020) International Committee of the Red Cross (ICRC): general guidance for the management of the dead related to COVID-19. Forensic Sci Int Synergy 2:129-137. https:// doi.org/10.1016/j.fsisyn.2020.03.007

4. Kanchan T, Saraf A, Krishan K, Misra S (2020) The advantages of virtopsy during the COVID-19 pandemic. Med Leg J. https://doi. org/10.1177/0025817220943035

5. Uchigasaki S (2006) Postmortem ultrasound imaging in forensic pathology. In: Tsokos M (ed) Forensic pathology reviews-book series, 4th edn. Humana Press, Totowa

6. Jackson K, Butler R, Aujayeb A (2020) Lung ultrasound in the COVID-19 pandemic. Postgrad Med J. https://doi.org/10.1136/postg radmedj-2020-138137

7. Peng QY, Wang XT, Zhang LN, Chinese Critical Care Ultrasound Study Group (CCUSG) (2020) Findings of lung ultrasonography of novel corona virus pneumonia during the 2019-2020 epidemic. Intensive Care Med 46(5):849-850. https://doi.org/10.1007/s0013 4-020-05996-6

8. Fariña J, Millana C, Fdez-Aceñero MJ et al (2002) Ultrasonographic autopsy (echopsy): a new autopsy technique. Virchows Arch 440(6):635-639. https://doi.org/10.1007/s00428-002-0607-z

9. Charlier P, Chaillot PF, Watier L, Ménétrier M, Carlier R, Cavard $S$ et al (2013) Is post-mortem ultrasonography a useful tool for forensic purposes? Med Sci Law 53(4):227-234. https://doi. org/10.1177/0025802413479946

Publisher's Note Springer Nature remains neutral with regard to jurisdictional claims in published maps and institutional affiliations. 